ARTICLE

\title{
Tunable unidirectional nonlinear emission from transition-metal-dichalcogenide metasurfaces
}

\author{
Mudassar Nauman (1) 1,2, Jingshi Yan², Domenico de Ceglia ${ }^{3 凶}$, Mohsen Rahmani id 4 , \\ Khosro Zangeneh Kamali [D ${ }^{2}$, Costantino De Angelis ${ }^{5}$, Andrey E. Miroshnichenko (1D ${ }^{6 凶}$, Yuerui Lu (DD ${ }^{1 凶} \&$ \\ Dragomir N. Neshev (iD ${ }^{2 凶}$
}

\begin{abstract}
Nonlinear light sources are central to a myriad of applications, driving a quest for their miniaturisation down to the nanoscale. In this quest, nonlinear metasurfaces hold a great promise, as they enhance nonlinear effects through their resonant photonic environment and high refractive index, such as in high-index dielectric metasurfaces. However, despite the sub-diffractive operation of dielectric metasurfaces at the fundamental wave, this condition is not fulfilled for the nonlinearly generated harmonic waves, thereby all nonlinear metasurfaces to date emit multiple diffractive beams. Here, we demonstrate the enhanced single-beam second- and third-harmonic generation in a metasurface of crystalline transition-metaldichalcogenide material, offering the highest refractive index. We show that the interplay between the resonances of the metasurface allows for tuning of the unidirectional secondharmonic radiation in forward or backward direction, not possible in any bulk nonlinear crystal. Our results open new opportunities for metasurface-based nonlinear light-sources, including nonlinear mirrors and entangled-photon generation.
\end{abstract}

\footnotetext{
${ }^{1}$ School of Engineering, Australian National University, Canberra, ACT 2601, Australia. ${ }^{2}$ ARC Centre of Excellence for Transformative Meta-Optical Systems, Department of Electronic Materials Engineering, Research School of Physics, The Australian National University, Canberra, ACT 2601, Australia.

${ }^{3}$ Department of Information Engineering, University of Padova, Via G. Gradenigo, 6/B Padova, Italy. ${ }^{4}$ Advanced Optics and Photonics Laboratory, Department of Engineering, School of Science and Technology, Nottingham Trent University, Nottingham NG11 8 NS, UK. ${ }^{5}$ Department of Information Engineering, University of Brescia, Via Branze 38, 25123 Brescia, Italy. ${ }^{6}$ School of Engineering and Information Technology, University of New South Wales, Canberra, ACT 2600, Australia. ${ }^{凶}$ email: domenico.deceglia@unipd.it; andrey.miroshnichenko@unsw.edu.au; yuerui.lu@anu.edu.au;

dragomir.neshev@anu.edu.au
} 
$\mathrm{N}$ onlinear optical phenomena in nanostructured materials have recently attracted much attention due to their wide spectrum of possible applications ${ }^{1,2}$ ranging from sensing to novel light sources. In these studies, the enhancement of the nonlinear processes at the nanoscale has been a key enabler. The two main paths in this development have been the use of highquality factor resonant photonic structures and highly nonlinear materials, including high refractive-index materials following the so-called Miller's rule. The earlier approaches were based on the use of plasmonic nanoantennas and metasurfaces ${ }^{3-6}$ as well as multiple-quantum-well (MQW) metasurfaces ${ }^{7-9}$. However, the high dissipative losses of plasmonic materials and the mid-infrared operation wavelengths of MQWs metasurfaces limit their broad applicability. More recently, high-index dielectric nanostructures have opened a new paradigm for nonlinear metaoptics $^{10,11}$. The excitation of Mie-type resonances in such nanostructures plays a vital role in enhancing the light-matter interactions, such as nonlinear harmonic generation ${ }^{12-14}$. The use of high-index dielectric materials, such as semiconductors, have simultaneously provided a strong intrinsic nonlinear susceptibility and high-quality factor resonances. Several pioneering works prompted the use of nanostructures from materials with established fabrication to enhance the process of third-harmonic generation (THG). Among them, $\mathrm{Si}^{15-17}$ and $\mathrm{Ge}^{18}$ show the high refractive index in the near-infra-red spectral range and exhibit strong nonlinear responses. However, Si and Ge are centrosymmetric materials and thereby inhibit the second-order nonlinear effects ${ }^{7}$.

Conversely, zinc-blende III-V semiconductors, such as GaAs and $\mathrm{AlGaAs}$ have received a great deal of interest for nonlinear metasurfaces due to their high-refractive indices and strong quadratic susceptibilities ${ }^{19-25}$. However, zinc-blende III-V materials are not transparent in the visible range and nanostructures are difficult to fabricate on low-index transparent substrates. Further such materials exhibit a peculiar nonlinear susceptibility with only off-diagonal non-zero $\chi^{(2)}$ tensor components ${ }^{20}$. As a consequence, in (100) AlGaAs metasurfaces, the second-harmonic generation (SHG) is inhibited at the normal direction and the harmonic emission is directed into the diffraction orders ${ }^{26,27}$. This nonlinear diffraction phenomenon limits the possible applications of metasurfaces as nonlinear light sources, where a single beam of emission is required. While metasurfaces made of inversion-symmetry-broken nanoresonators ${ }^{28,29}$ and individual nanoantennas ${ }^{30,31}$ have been proposed to direct the emission at the normal direction, to date they operate in the diffractive regime ${ }^{12-14}$, with multiple diffractive orders being emitted at the harmonic waves ${ }^{27,32}$. The ability to operate in the zero-order SHG and THG, as well as to be able to tune the directionality of such unidirectional nonlinear emission from forward to backward direction remains elusive.

Here, we demonstrate the significant enhancement of SHG and THG in a transition-metal-dichalcogenide (TMDC) $\mathrm{MoS}_{2}$ metasurface in the sub-diffractive, single-beam nonlinear emission in the visible to the near-infra-red regime. Our metasurfaces consist of nanoresonators with the highest refractive index $>4$, in the visible range, prompting strong material nonlinearity. The high refractive index further allows for optical resonances in smallersize nanoresonators, thereby for densely packed metasurfaces. In particular, we design our metasurface to exhibit multipolar resonances at the second-harmonic wavelengths and employ the interference of this resonance to enable the tuning of the unidirectional emission of the second-harmonic light in forward or backward direction, controlled either by the excitation wavelength or by the incident polarisation. In all operation regimes, our metasurface remains sub-diffractive for the SHG and THG wavelengths. Finally, our TMDC metasurface can be fabricated on any transparent substrate due to the strong van der Waals (vdW) stiction forces of the layered $\mathrm{MoS}_{2}$. As such, our TMDC metasurfaces provide a versatile tool to tune all harmonic emissions and open new applications of nonlinear metasurfaces for advanced light sources ${ }^{33}$ or nonlinear mirrors ${ }^{34}$.

\section{Results}

Linear response. TMDCs are vdW materials of high refractive index with a relatively large, indirect bandgap in the visible spectrum. They have attracted significant attention for highperformance photonic devices, such as miniaturised lenses ${ }^{35,36}$ and gratings ${ }^{37}$. More recently, TMDCs have sparked an interest in novel dielectric metasurfaces with high refractive index ${ }^{38-44}$ due to the opportunity to fabricate high purity single-crystalline films on any transparent substrate. The TMDC materials, such as $\mathrm{MoS}_{2}$ further exhibit low optical losses in the visible and near infra-red spectral regions. The refractive index of the $\mathrm{MoS}_{2}$ is over 4.5 (Supplementary Fig. 1 of Supplementary Information) in the region $700-800 \mathrm{~nm}$, which is significantly higher than in other nonlinear metasurfaces, e.g. those based on $\mathrm{Si}$, GaAs or AlGaAs, and promotes strong nonlinear response.

Here, we fabricate a TMDC-based $\mathrm{MoS}_{2}$ metasurface, which supports optically induced resonant modes in the visible spectrum, where $\mathrm{MoS}_{2}$ has a negligible absorption. According to Mie theory ${ }^{10}$, these resonances depend upon the refractive index and the geometric parameters of the individual metaatoms ${ }^{45}$. We start with exfoliating high-quality multilayer $\mathrm{MoS}_{2}$ flakes on a sapphire substrate. The flake is then patterned using electron beam lithography (EBL) and reactive-ion etching (see "Methods"). The design of our nonlinear metasurface is illustrated in Fig. 1a. It consists of a periodic square array of truncated cone meta-atoms, placed on low refractive index sapphire $\left(\mathrm{Al}_{2} \mathrm{O}_{3}\right)$ substrate $(\sim 500 \mu \mathrm{m}$ thick $)$. A scanning electron microscopy (SEM) image of a typical metasurface is shown in Fig. 1b. Metasurfaces of three different geometric dimensions have been fabricated $(\mathrm{A}-\mathrm{C})$, exhibiting resonances from the visible to the near-infrared spectral range.

The linear transmission spectra of the fabricated metasurfaces (A-C having the same height $\cong 150 \mathrm{~nm}$ but different radii $100 \mathrm{~nm}, 110 \mathrm{~nm}, 120 \mathrm{~nm}$, respectively) were measured in a custom-built white light transmission spectroscopy setup and are shown in Fig. 1c. The white light is focused onto a sample and collected by $20 \times$ microscope objective lenses $(\mathrm{NA}=0.4)$. The spectra are recorded by two different spectrometers for the visible and infra-red parts of the spectrum, respectively. All three metasurfaces show distinct Mie-type resonances in the spectral range of $700-800 \mathrm{~nm}$. We have also performed numerical simulations (Lumerical FDTD) to systematically study the resonant modes of the fabricated $\mathrm{MoS}_{2}$ metasurfaces (Supplementary Fig. 1 of Supplementary Information). The induced resonances are associated with transmittance dips and are marked by arrows in Fig. 1c. By changing the aspect ratio (periodicity is changed together with the diameter of meta-atoms, whilst keeping the height unchanged) of the $\mathrm{MoS}_{2}$ truncated cones (for metasurfaces A-C) we can control the spectral position of the resonances from visible to infra-red wavelengths ${ }^{46}$. All metasurfaces are fully transparent at a longer wavelength $(>900 \mathrm{~nm})$ (Supplementary Fig. 2 of Supplementary Information).

The nature of the resonances can be further verified by examining the cartesian multipole expansion of the near fields of metasurface C. The linear scattering is illustrated in Fig. $1 \mathrm{~d}$ by the effective scattering cross-section, $\sigma$ (black curve) and the corresponding transmittance through the metasurface. A qualitative agreement is observed between the measured and simulated results (Supplementary Fig. 1 of Supplementary Information). 


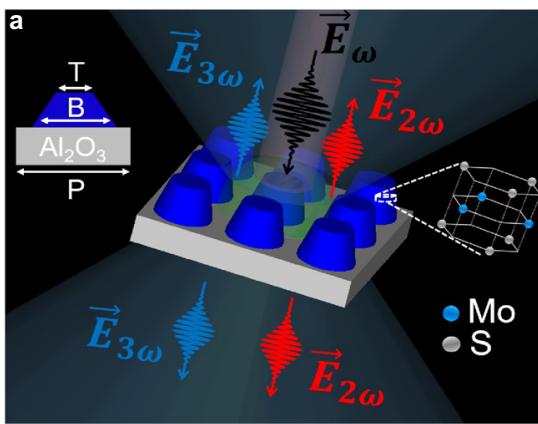

d
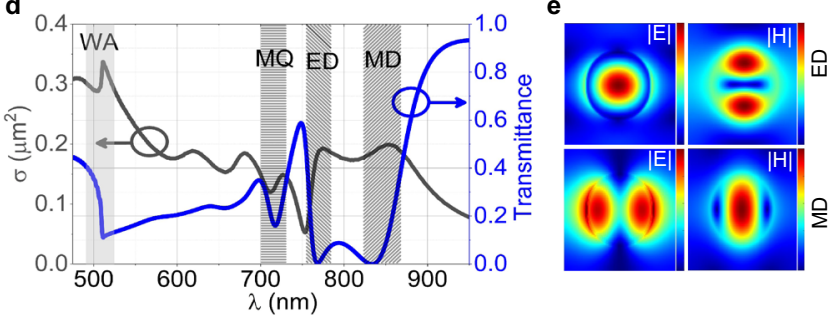

Fig. 1 Nonlinear TMDC metasurfaces. a Schematic of $\mathrm{MoS}_{2}$ truncated cone metasurface. For metasurface $C, T=150 \mathrm{~nm}, \mathrm{~B}=240 \mathrm{~nm}, \mathrm{P}=300 \mathrm{~nm}$ and height $=150 \mathrm{~nm}$, as shown in the top left corner. The bottom-right inset shows the lattice structure of the $2 \mathrm{H}$-stacked $\mathrm{MoS}_{2}$. b The SEM image of the fabricated metasurface $C$ with a scale bar of $2 \mu \mathrm{m}$ while the inset scale bar is $500 \mathrm{~nm}$. c The measured transmission spectrum of three metasurfaces A-C. For clarity, spectra are vertically displaced by $T=1$ for each metasurface. Arrows show the change in the spectral position of electric dipole (ED) and magnetic dipole (MD) as a function of the aspect ratio of truncated cone meta-atoms. d Calculated cartesian multipoles expansion of metasurface $\mathrm{C}$, where $\sigma$ represents the effective scattering cross-section. The patterned regions represent the position of magnetic quadrupole (MQ), ED and MD, whilst the light grey region shows the position of first-order Wood's anomaly WA. e Normalised electric and magnetic field profiles at the resonant wavelengths: $770 \mathrm{~nm}$ and $840 \mathrm{~nm}$, respectively.

This agreement further allows us to identify the nature of the resonances by exploring the symmetry of the electric and magnetic fields. These are plotted in Fig. 1e, showing the distributions of electric and magnetic fields in a horizontal cutplane through the centre of the truncated cone meta-atom. The resonance mode at shorter wavelengths has a maximum of the electric field in the centre of the resonator and correspond to an electric dipole (ED) mode. The longer wavelength resonance mode has, in contrast, maximum of the magnetic field in the centre of the resonator and is linked to a magnetic dipole (MD) mode. In addition, all metasurfaces support higher-order Mietype resonances, for wavelengths below $700 \mathrm{~nm}$. These higherorder resonances, however, fall in the region where the $\mathrm{MoS}_{2}$ possess excitonic resonances and higher absorption, see also the $\mathrm{MoS}_{2}$ dispersion in Supplementary Fig. 1 of Supplementary Information. Thereby, experimentally these high-order Mie resonances are difficult to identify and are not marked in the experimental spectrum, Fig. 1c. Nevertheless, for metasurface C the magnetic quadrupole (MQ) mode can be seen as a small shoulder around $715 \mathrm{~nm}$, in Fig. 1c. The total extinction crosssection obtained by multipolar decomposition in Fig. 1d shows dominant $\mathrm{ED}, \mathrm{MD}$ and MQ contributions of the resonances.

However, all resonant modes are generally of mixed nature and to better identify their character we further performed quasinormal mode (QNM) theory simulations ${ }^{47}$ for metasurface C. The QNM representation allows us to quantify the coupling of the modes to free space and determine the role and weight of the individual modes into the harmonic radiation. To retrieve the characteristic wavelength and the lifetime of the QNM we consider the metasurface as an open-cavity system that supports eigenmodes and solve this complex eigenvalue (i.e., source-free) problem with finite-element numerical simulations (COMSOL). The eigenvalues of the problem are the QNMs complex eigenfrequencies $\tilde{\omega}_{m}=\operatorname{Re}\left(\tilde{\omega}_{m}\right)+i \operatorname{Im}\left(\tilde{\omega}_{m}\right)$, the real part being associated with the characteristic wavelengths $\tilde{\lambda}_{m}=2 \pi c / \operatorname{Re}\left(\tilde{\omega}_{m}\right)$ of the modes and the imaginary part associated with the modes lifetimes $\tilde{\tau}_{m}=1 / \operatorname{Im}\left(\tilde{\omega}_{m}\right)$. The knowledge of the eigenvalues $\tilde{\omega}_{m}$ and the normalised eigenfields $\tilde{\mathbf{E}}_{\mathrm{m}}$ associated with QNMs, allows decomposing the radiation of the metasurface at these wavelengths, such as the SHG, as follows

$$
\mathbf{E}_{\mathrm{rad}}=\sum_{m} \alpha_{m} \tilde{\mathbf{E}}_{\mathrm{m}}
$$

where the weighting factors, $\alpha_{m}$, are frequency-dependent functions proportional to the overlap integrals of the eigenmodes with the background field $\mathbf{E}_{b}$ generated by the source in the absence of the resonators (in our case, the system is composed simply by an interface between air and the sapphire substrate). The eigenfields are normalised as in ref. ${ }^{48}$. We further adopt the following formulation $\alpha_{m}=-\frac{\tilde{\omega}_{m}}{\widetilde{\omega}_{m}-\omega_{\text {rad }}} \int_{V} \tilde{\mathbf{E}}_{m} \cdot \mathbf{E}_{b} d V$, where $\omega_{\text {rad }}$ is the angular frequency of the radiation. Our analysis shows that the $\mathrm{MoS}_{2}$ metasurface supports three QNMs with strong field confinement in the range of wavelengths between 700 and $900 \mathrm{~nm}$. The eigenfields distributions associated with these eigenmodes are reminiscent of the field distributions of $\mathrm{MD}$, ED and MQ-type Mie modes, as shown in Fig. 2a-c. In particular, we find an eigenmode at $\tilde{\lambda}_{\mathrm{MD}}=840 \mathrm{~nm}$ with an MD-like eigenfield and lifetime $\tau_{\mathrm{MD}}=6.7 \mathrm{fs}$. The second eigenmode at $\tilde{\lambda}_{\mathrm{ED}}=770 \mathrm{~nm}$ has an ED-like eigenfield and lifetime $\tau_{\mathrm{ED}}=21 \mathrm{fs}$. The third eigenmode at $\tilde{\lambda}_{\mathrm{MQ}}=715 \mathrm{~nm}$ has an MQ-like eigenfield and lifetime $\tau_{\mathrm{MQ}}=17 \mathrm{fs}$. In Fig. $2 \mathrm{~d}$, we show, for the same metasurface, the transmission spectrum for plane-wave excitation at normal incidence. The spectrum displays three transmission dips very close to the real eigen-wavelengths of the three QNMs. The signatures of the excitation of the three QNMs are the Fanolike resonances labelled as MD, ED and MQ. Such labels for the QNMs are only introduced to underscore the similarity of the eigenfields to the typical field distributions of multipolar modes. The ED, MD and MQ-like QNMs supported by the metasurface play a crucial role not only in the linear response (Fig. 2d) but also in enhancing the second-order nonlinear response obtained when the pump is tuned at double those eigen-wavelengths, as discussed in the next section.

Nonlinear response of TMDC metasurfaces. Next, we test the nonlinear frequency conversion from the TMDC metasurface. The optical microscope image of metasurface $\mathrm{C}$ is shown in Fig. 3a. We specifically chose the top-right corner of the metasurface, which shows a fabrication defect. Such a defect, however, allows for the spatial identification of the nonlinear emission from the metasurface. Next, we illuminate the metasurface by 10 ps laser pulses from a fibre laser and amplifier (Pritel, a repetition rate of $20 \mathrm{MHz}$ ), operating at a telecommunication wavelength of $1550 \mathrm{~nm}$. The pump is focused onto the sample by a $100 \times$ microscope objective $(\mathrm{NA}=0.7)$, and the nonlinear signal is collected in transmission. The measured power for the $\mathrm{SH}$ and TH signals shows quadratic and cubic dependence on the incident power, respectively, as shown in Fig. 3b. No saturation of the emitted $\mathrm{SH}$ and $\mathrm{TH}$ signals is observed in Fig. $3 \mathrm{~b}$ when the pump average power is increased up to $24 \mathrm{~mW}$, corresponding to a peak pump power of $120 \mathrm{~W}$ (peak intensity of $\sim 0.95 \mathrm{GW} / \mathrm{cm}^{2}$ ). The 

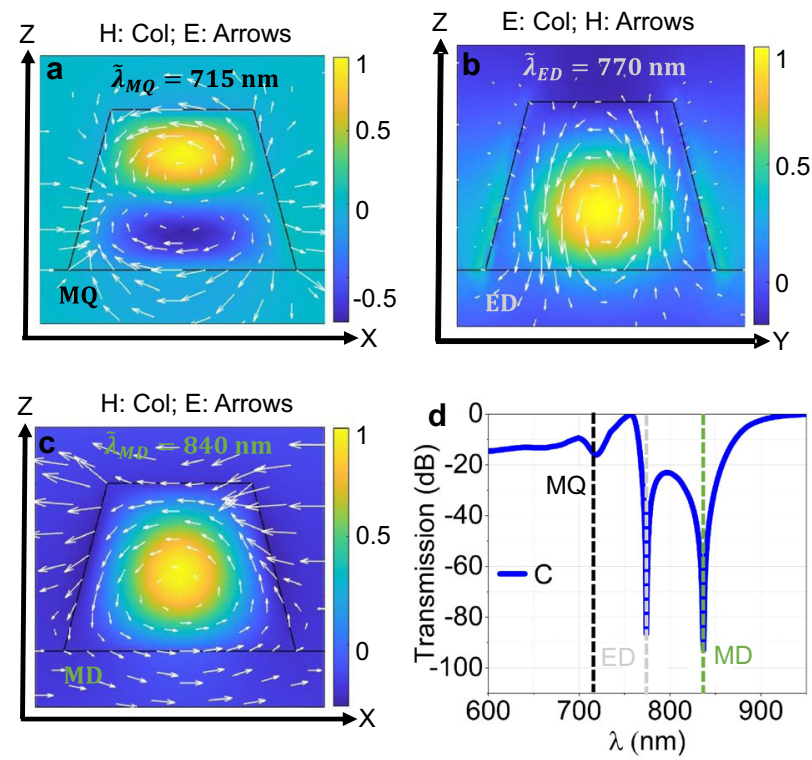

Fig. 2 Quasi-normal modes for metasurface C. a Eigenfields (electric field $\mathbf{E}$ and magnetic field $\mathbf{H}$ distribution) of the eigenwavelength $\left(\tilde{\lambda}_{\mathrm{MQ}}\right)$ is reminiscent of magnetic quadrupole (MQ) mode, E: shown as arrows and $\mathbf{H}$ : shown as colour. $\mathbf{b}$ Eigenfields (electric field $\mathbf{E}$ and magnetic field $\mathbf{H}$ distribution) of the eigenwavelength $\left(\tilde{\lambda}_{\mathrm{ED}}\right)$ is reminiscent of electric dipole (ED) mode, $\mathbf{E}$ : shown as arrows and $\mathbf{H}$ : shown as colour. $\mathbf{c}$ Eigenfields (electric field $\mathbf{E}$ and magnetic field $\mathbf{H}$ distribution) of the eigenwavelength $\left(\tilde{\lambda}_{\mathrm{MD}}\right)$ is reminiscent of magnetic dipole (MD) mode, E: shown as arrows and $\mathbf{H}$ : shown as colour. d Simulated transmission spectra calculated for plane-wave excitation at normal incidence for metasurface $\mathrm{C}$. Very close to the real eigenwavelengths (shown by vertical dashed lines, following the colour coding scheme of the eigenwavelengths shown in (a-c) we have three transmission dips. The Fano-like nature of these three transmission dips (around 715, 770, and $840 \mathrm{~nm}$ ) is a clear indication of the excitation of the three quasi-normal modes QNMs. As the eigenfields of these QNMs are reminiscent of the typical field distribution of $M Q, E D$ and $M D$ modes and for this reason, we have labelled the three QNMs as "MQ", "ED" and "MD" at the bottom inset of a-c, respectively.

sample is embedded in a confocal scanning microscope (WITec) that allows for performing nonlinear imaging microscopy by scanning the sample stage. The THG and SHG nonlinear images of the $\mathrm{MoS}_{2}$ metasurface $\mathrm{C}$ are shown in Fig. 3c, d, respectively. The obtained THG $(517 \mathrm{~nm})$ and SHG $(775 \mathrm{~nm})$ signals are at wavelengths where the metasurface exhibits the first-order WA in the substrate and the ED-like QNM, respectively. We note that the third harmonic is significantly enhanced by the metasurface, well above the background signal from the substrate. The second harmonic response of the bulk $\mathrm{MoS}_{2}$ depends upon the number of layers in the $\mathrm{MoS}_{2}$ flake. A flake with an odd number of layers is non-centrosymmetric and it belongs to $\mathbf{D}_{3 \mathbf{h}}^{1}$ space group $^{49,50}$. A $\mathrm{MoS}_{2}$ flake with an even number of layers is centrosymmetric and belongs to $\mathbf{D}_{3 \mathbf{d}}^{3}$ space group ${ }^{49}$ thereby it does not exhibit secondorder nonlinaerity ${ }^{49,51}$. However, the metasurface appears to result in a moderate amount of SHG, again well above the background of the substrate. While recent works ${ }^{41}$ suggest that the SHG from TMDC nanoresonators has a bulk response, such possibility is unlikely and requires mode detailed analyses, as shown below.

It is worth noting that to provide a sub-diffractive nonlinear response, the periodicity of the metasurface should be, $P<\lambda / n$, where $P$ is the metasurface periodicity, $n$ is the refractive index of the surrounding environment, being either air or sapphire substrate in our case, and $\lambda$ is the wavelength of the harmonic
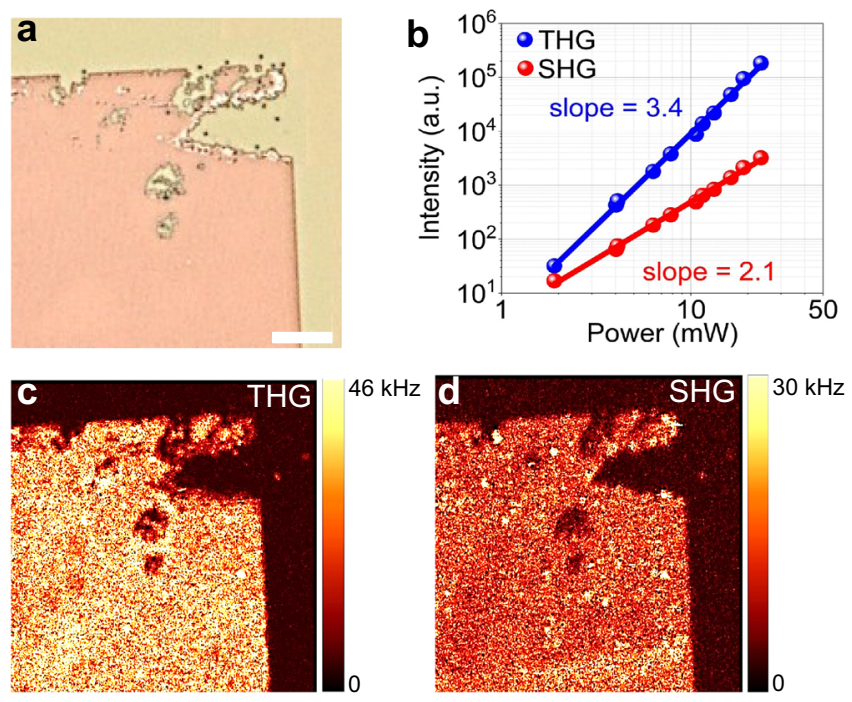

Fig. 3 Nonlinear microscopy of TMDC metasurfaces. a Optical microscopic image of $\mathrm{MoS}_{2}$ truncated cone metasurface $\mathrm{C}$, scale bar is $10 \mu \mathrm{m}$. b Power dependent nonlinear second harmonic generation SHG, red colour, and third harmonic generation THG, blue colour, signals from the pump laser at $1550 \mathrm{~nm}$. c, d Third- and second-order nonlinear spatial mapping of the metasurface $C$, respectively.

wave (THG or SHG). This condition stems from the equation for the diffraction angle $\theta_{\text {diff }}$,

$$
\theta_{\text {diff }}=\sin ^{-1} \frac{\lambda_{\mathrm{SH}}}{P \times n} .
$$

As compared to other lower-index semiconductor metasurfaces the use of the high-index TMDCs allows us to design highly subwavelength resonators and therefore to construct nonlinear metasurfaces with sub-diffractive properties at the harmonic waves.

THG in TMDC metasurfaces. First, we examine the enhancement of the THG process in the TMDC metasurface. For this purpose, we use a tunable femtosecond laser (Chameleon Ultra II and OPO, pulse width of $\sim 200 \mathrm{fs}$ ) in a home-build microscopy setup. We performed wavelength-dependent nonlinear measurements by focusing the laser beam onto the sample using a $20 \times$ microscope objective $(\mathrm{NA}=0.4)$. We then collect the third harmonic in the forward direction by a second objective $(100 \times$, $\mathrm{NA}=0.7$ ). To filter out the transmitted and reflected pump wave, we employed a short-pass filter at $800 \mathrm{~nm}$. Two waveplates are used to control the polarisation of the pump beam. Figure $4 \mathrm{a}$ depicts the spectra of the THG from metasurface $C$ for different pump wavelengths. The black curve represents a spline through all maxima. Two distinct regions could be observed in THG: below and above the $\mathrm{WA}^{52}$ marked with grey shading in Fig. $4 \mathrm{a}$. Below the WA, our metasurface operates in the diffractive regime, where a large amount of the THG is emitted into the diffraction orders and not collected by the microscope objective and the detector. At the WA, the third harmonic is emitted in the plane of the metasurface and no light is detected. For wavelengths above the WA, the metasurface is sub-diffractive, and the THG is emitted into the zeroth-order only, which shows a pronounced enhancement at $517 \mathrm{~nm}$. Measurements above $530 \mathrm{~nm}(>1590 \mathrm{~nm}$ pump wavelength) are unfortunately not accessible by our pump laser. In our experiments, the highest third harmonic power is obtained at a wavelength of $1550 \mathrm{~nm}$, resulting in THG efficiency of $1.01 \times 10^{-9}$ at peak power of $4.3 \mathrm{~kW}$, beam full width at half maximum (FWHM) of $4.6 \mu \mathrm{m}$, see Supplementary Fig. 3 of Supplementary Information. While the absolute efficiency is 

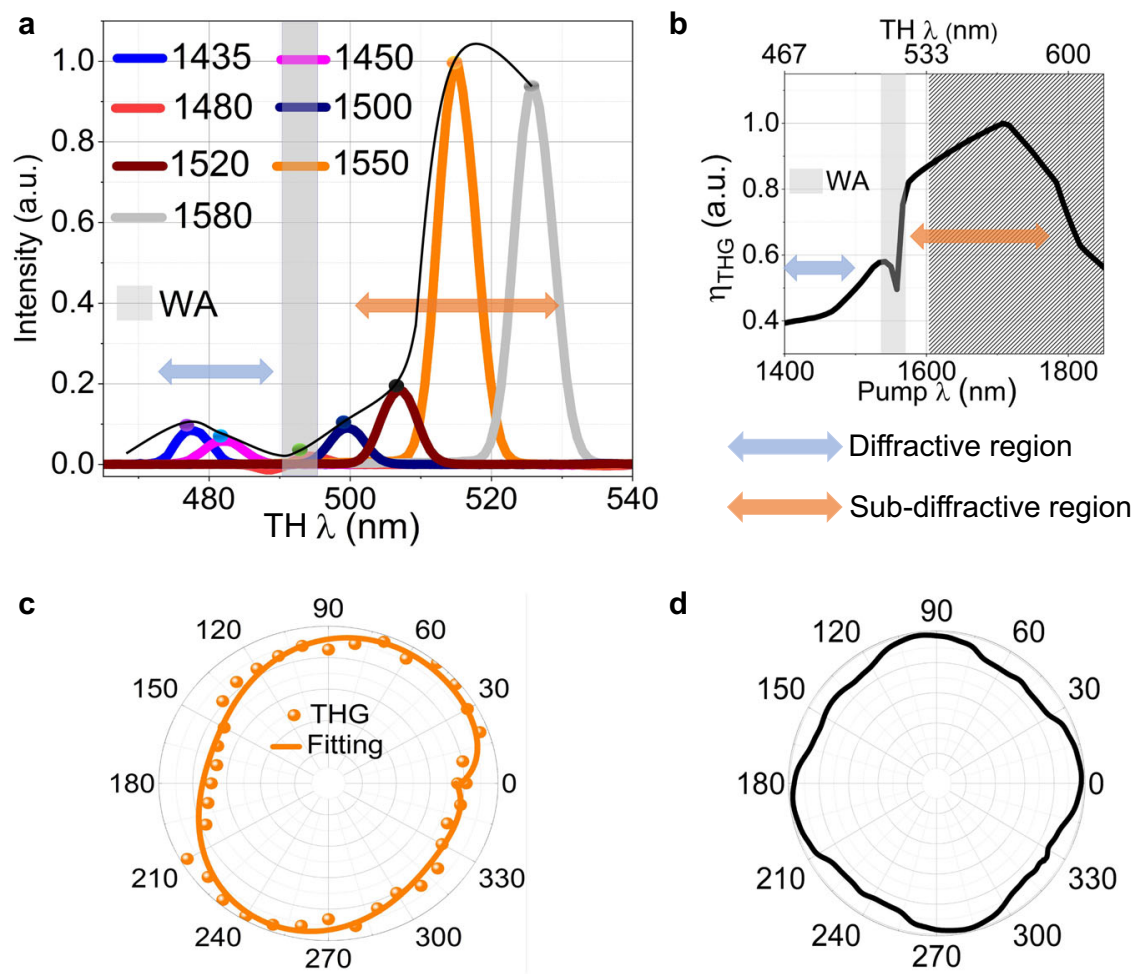

Fig. 4 Third harmonic generation enhancement in a TMDC metasurface. a Measured wavelength-dependent third harmonic response in forward direction for metasurface $C$. The shaded region represents Wood's anomaly WA. b Simulated third harmonic conversion efficiency $\eta_{T H G}$, as a function of pump wavelength, of $\mathrm{MoS}_{2}$ truncated cone metasurface $\mathrm{C}$. The patterned region represents the spectrum range not accessible by the laser in our experimental setup. c Experimental polarisation resolved third harmonic generation THG of metasurface C, at a pump wavelength of $1550 \mathrm{~nm}$. $\mathbf{d}$ Simulated polarisation resolved THG of metasurface C, pump wavelength $1550 \mathrm{~nm}$.

lower than the previous works on THG in single silicon nanostructures, the current value is obtained at five times lower intensity. Considering the quadratic dependence of the efficiency on the intensity and the lack of resonances at the fundamental wavelengths, the obtained value is impressive. In Fig. 4a, the THG intensity is normalised to the cubic power of the incident beam intensity, to avoid the small variations of the incident power, while tuning the OPO wavelength.

To gain a better insight on the physics of the third harmonic emission, we further perform full-wave numerical simulations of the THG in our metasurface. Figure $4 \mathrm{~b}$ shows the simulated conversion efficiency of THG as a function of pump wavelength. The simulated THG conversion efficiency of metasurfaces A and B is presented in Supplementary Fig. 4 of Supplementary Information. The numerical data, Fig. 4b, shows good qualitative agreement with the experimental data in Fig. 4a, capturing the two main effects: (i) There is a peak of the THG at longer wavelengths in the non-diffractive regime, at a pump wavelength of approximately $1700 \mathrm{~nm}$. (ii) Both in the experiment and in the simulations, a drastic decrease of the TH efficiency is observed at and below the WA. We note that the higher-order Mie modes, supported by the metasurface at the third harmonic wavelengths are hindered by absorption losses. While $\mathrm{MoS}_{2}$ has a very high refractive index in the spectral region $450-600 \mathrm{~nm}(n=4.8-5.6)$, it also has a large absorption coefficient $(k=3.1-1.1)$.

Figure $4 \mathrm{c}, \mathrm{d}$ further shows the experimentally measured and simulated polarisation resolved THG response of the metasurface $\mathrm{C}$ at $517 \mathrm{~nm}$ (pump wavelength $1550 \mathrm{~nm}$ ). This response reveals that the THG power is nearly independent of the incident polarization and co-linearly polarised with the pump beam. Good agreement is observed between our experiment and simulations. This observation confirms that the crystallinity of the $\mathrm{MoS}_{2}$ metasurface belongs to the $\mathrm{D}_{3 \mathrm{~h}}$ symmetry group. The slight asymmetry in the experimental polarisation measurement is likely caused by a slight misalignment in the experimental setup and slight asymmetry in the fabricated samples.

Furthermore, full-wave nonlinear simulations have been performed in order to investigate the tapering angle effect of the following three meta-atom shapes: (i) cone, (ii) truncated cone, (iii) vertical cylinder, on the THG conversion efficiency (in either direction forward and backward) and the results are presented in Supplementary Fig. 5 of Supplementary Information. The THG directionality is quite similar in truncated shapes, cone and truncated conical meta-atoms. Whilst larger THG directionality is observed, in the entire spectrum (1400-1600 nm), in vertical straight cylindrical meta-atoms because of their large volume as compared to truncated meta-atom shapes.

Second-harmonic generation in TMDC metasurfaces. Next, we turn our attention to the second-order nonlinear response of the TMDC metasurfaces and study the resonant enhancement and the directionality of the SHG. The $\mathrm{MoS}_{2}$ metasurface supports several resonant QNMs at the $\mathrm{SH}$ wavelengths, as indicated in Fig. 2. The coupling of SH light to these modes boost the SHG efficiency and promotes its radiation to the far-field ${ }^{33}$.

To test the influence of the resonant modes on the $\mathrm{SH}$ enhancement, we examined the wavelength dependence of the SHG from metasurface C. For this purpose, we scan the wavelength of the infra-red pump beam in the range of $1200-1600 \mathrm{~nm}$. The measured SH spectra for different pump wavelengths are shown in Fig. 5a. Two peaks of SH emissions can be observed when the $\mathrm{SH}$ wavelength is in the vicinity of the MQ and ED QNMs. The SH enhancement, however, is significantly higher at the position of the MQ mode at $1400 \mathrm{~nm}$, despite the 

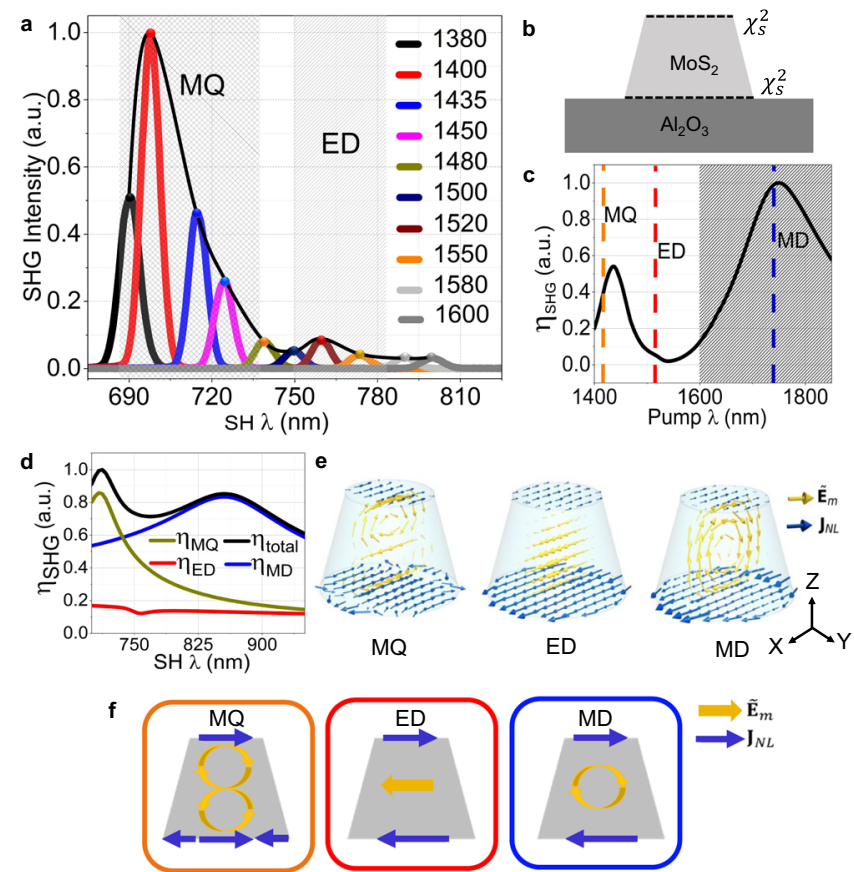

Fig. 5 Second harmonic generation in a TMDC metasurface. a

Experimentally measured wavelength dependence of the SHG from metasurface $\mathrm{C}$. The second harmonic generation (SHG) was enhanced in the vicinity of magnetic quadrupole (MQ) and electric dipole (ED). b Schematic showing the locations of the SH current source $\chi^{2}$. c Calculated second harmonic generation conversion efficiency $\left(\eta_{\mathrm{SHG}}\right)$ by employing fullwave nonlinear simulations. The patterned region is the spectral range not accessible by the laser in our experiments. d SHG conversion efficiency calculated using quasi-normal modes couple-mode theory. e Spatial overlap between the induced nonlinear current source $\mathbf{J}_{\mathrm{NL}}$ and eigenfields of the calculated quasi-normal modes $\tilde{\mathbf{E}}_{\mathrm{m}}$. $\mathbf{f}$ Schematic of the modal overlap of nonlinear current source and eigenfields.

stronger far-field scattering at the position of the ED mode. This feature of the $\mathrm{SH}$ enhancement can be explained by considering the near-field intensity enhancement, its overlap with the location of $\mathrm{SH}$ current sources and the modal overlap of the eigenmodes at the participating frequencies. The highest $\mathrm{SH}$ efficiency is estimated at $3.4 \times 10^{-10}$, at incident peak power of $4.3 \mathrm{~kW}$, beam FWHM of $4.6 \mu \mathrm{m}$, see Supplementary Fig. 3 of Supplementary Information.

While ref. ${ }^{41}$ treats the nanoresonators as bulk nonlinear media, the bulk $\mathrm{MoS}_{2}$ has $\mathrm{D}_{6 \mathrm{~h}}$ symmetry and does not exhibit bulk second-order nonlinear effects. However, for a few-layerthick material, the actual second-order nonlinear response will depend on the exact number of layers ${ }^{50}$. In the case of TMDC metasurfaces, the situation is even more complex due to the inhomogeneous near fields and the absorption of the $\mathrm{MoS}_{2}$. Therefore, it is important to quantify the position and origin of the nonlinear sources for SHG in TMDC metasurfaces.

To determine the location of the SH current sources, we assumed four extreme scenarios on how the second-order nonlinearity $\left(\chi^{(2)}\right)$ is present in TMDC metasurfaces. These include having nonlinear sources: (i) at top and bottom surfaces, (ii) in the bulk, (iii) at the top surface only and (iv) at the bottom surface only. To characterise these four cases, we performed fullwave nonlinear simulations of the metasurface SHG response. The analysis of these four scenarios is discussed in detail in the Supplementary Information (see Supplementary Fig. 6). Among these four scenarios, the first one best describes our experimental $\mathrm{SH}$ response. Namely, the $\mathrm{SH}$ response is a result of the interference of the SHG from the top and bottom layers of the $\mathrm{MoS}_{2}$, as depicted in Fig. 5b. This is in stark contrast to the surface SHG emission from nanostructures made of centrosymmetric materials, such as silicon metasurfaces ${ }^{54}$. In such nanostructures, the second harmonic emission originates mainly from their sidewalls, where the symmetry is broken. In our TMDC metasurface, the dominant contribution is from the top and bottom $\mathrm{MoS}_{2}$ layers, which are approximately four orders of magnitude more efficient.

The corresponding simulations for the wavelength dependence of the $\mathrm{SH}$ efficiency from metasurface $\mathrm{C}$ are presented in Fig. $5 \mathrm{c}$. A good agreement can be observed with the measured SHG (Fig. 5a). We note that our theoretical calculations show the strongest SHG at the position of the MD mode, which is not accessible by our tunable pump laser. To quantify this wavelength dependence, we further performed coupled-mode analysis on the nonlinear wave interactions. The SHG efficiency depends upon the coupling between the $\mathrm{SH}$ light and eigenmodes. It is well known that in nanostructured resonant systems, the SHG efficiency, which is the ratio of $\mathrm{SH}$ to pump power, depends on how large is the spatial field overlap between the pump and $\mathrm{SH}$ field in the regions occupied by nonlinear sources. Indeed, using the reciprocity theorem, a simple expression of the SHG efficiency can be obtained in terms of overlap integrals ${ }^{8}$

$$
\eta_{\mathrm{SHG}} \sim\left|\int_{S} \mathbf{J}_{\mathrm{NL}}(2 \omega) \cdot \mathbf{E}_{\mathrm{SH}} d S\right|,
$$

where $\mathbf{J}_{\mathrm{NL}}(2 \omega)=-i \omega_{\mathrm{SH}} \epsilon_{0} \chi_{\mathrm{MoS}_{2}}^{(2)}\left[\left(E_{p, x}^{2}-E_{p, y}^{2}\right) \hat{\boldsymbol{x}}-2 E_{p, x} E_{p, y} \hat{y}\right]$ is the nonlinear surface current source induced by the pump at the $\mathrm{SH}$ wavelength. The aforementioned expression of the $\mathbf{J}_{\mathrm{NL}}(2 \omega)$, that we consider in the model and in nonlinear simulations stems from the tensor nature of $\mathrm{MoS}_{2}$, which has the following nonzero elements: $\chi_{S}^{(2)}\left(\mathrm{MoS}_{2}\right)=\chi_{x x x}^{(2)}=-\chi_{x y y}^{(2)}=-\chi_{y x y}^{(2)}=-\chi_{y y x}^{(2)}$. $\mathbf{E}_{\mathrm{SH}}$ is the field-induced on the surface of the resonator by a far-field virtual source, i.e., a plane wave located at the detector's position. The integral is performed over the top and bottom surfaces of the $\mathrm{MoS}_{2}$ meta-atoms. The pump field, $\mathbf{E}_{\mathrm{p}}$, and the $\mathrm{SH}$ field, $\mathbf{E}_{\mathrm{SH}}$, can be retrieved by solving the linear problems with a plane-wave excitation at the pump wavelength. Since in our problem the modes are resonantly excited at the SH wavelength, we only expand the SH field in QNMs, and we retain only the three QNMs that are present in the $\mathrm{SH}$ spectrum under investigation, i.e., the $\mathrm{MD}$, the $\mathrm{ED}$ and the $\mathrm{MQ}$-like modes.

The metasurface response at the pump wavelengths $(1200-1600 \mathrm{~nm})$ is non-resonant, and we can therefore retain the full-wave expression of $\mathbf{J}_{\mathrm{NL}}(2 \omega)$ without expanding the pump field $\mathbf{E}_{\mathrm{p}}$. As a result, the expression of the SHG efficiency can be recast as follows:

$$
\begin{aligned}
\eta_{\mathrm{SHG}} \sim & \mid \alpha_{\mathrm{MD}} \int_{S} \mathbf{J}_{\mathrm{NL}}(2 \omega) \cdot \tilde{\mathbf{E}}_{\mathrm{MD}} d S+\alpha_{\mathrm{ED}} \int_{S} \mathbf{J}_{\mathrm{NL}}(2 \omega) \cdot \tilde{\mathbf{E}}_{\mathrm{ED}} d S \\
& +\alpha_{\mathrm{MQ}} \int_{S} \mathbf{J}_{\mathrm{NL}}(2 \omega) \cdot \tilde{\mathbf{E}}_{\mathrm{MQ}} d S \mid .
\end{aligned}
$$

The expression in Eq. (4) is only based on the linear properties of the metasurface and is limited to only three QNMs. As such, it does not give an exact quantitative description of the SHG efficiency, however, it provides useful insights into the origin of SH light in our system. In particular, Eq. (4) predicts the presence and the nature of SHG spectral peaks and allows to quantify the coupling strength of SH light to each QNM. Furthermore, Eq. (4) can be used to optimise the meta-atom shape and size in order to enhance or inhibit the response of specific QNMs or to favour the 
interaction between different QNMs (see Supplementary Fig. 7 of Supplementary Information).

In Fig. 5d, we also show the calculated SHG conversion efficiency using the coupled QNM theory, Eq. (4), as well as the contributions of each of the three QNMs to the overall efficiency

$$
\eta_{m}=\left|\alpha_{m} \int_{S} \mathbf{J}_{N L}(2 \omega) \cdot \tilde{\mathbf{E}}_{m} d S\right|,
$$

where $m=\mathrm{MD}, \mathrm{ED}, \mathrm{MQ}$. The calculation is performed by considering a pump field, linearly polarised along the $x$-axis and the co-polarised component of the SHG. We can clearly observe that the SHG displays peaks of efficiency that are associated only with the two magnetic modes, while SH light coupling with ED-like mode is significantly inhibited. This result is in qualitative agreement with the full-wave nonlinear numerical simulations (Fig. 5c) and our experimental observations (Fig. 5a).

The reason behind the dominant role of magnetic modes over the electric mode is better clarified by inspecting the overlap integrals that appear in the expressions of $\eta_{m}$. In Fig. 5e, we show the spatial distribution of the eigenmodes $\tilde{\mathbf{E}}_{\mathrm{m}}$ along with the induced nonlinear surface currents $\mathbf{J}_{\mathrm{NL}}$. When the $\mathrm{SH}$ is tuned to the wavelengths of the magnetic QNMs, $\tilde{\lambda}_{\mathrm{MD}}$ and $\tilde{\lambda}_{\mathrm{MQ}}$, the spatial overlap between the nonlinear source and the eigenfield is optimal on both the top and bottom surface (see MQ and MD in Fig. 5e). On the contrary, this overlap is very weak for the ED mode (see ED in Fig. 5e). Although the ED mode displays strong field localisation at the centre of the meta-atom's volume, the eigenfield is less intense at the top and bottom surfaces, where the nonlinearity is present. In addition, the $\mathrm{SH}$ coupling to the $\mathrm{ED}$ mode is frustrated by the fact that the nonlinear current source $\mathbf{J}_{\mathrm{NL}}$ at $\tilde{\lambda}_{\mathrm{ED}}$ has odd parity, while the eigenfield $\tilde{\mathbf{E}}_{\mathrm{ED}}$ has even parity. This difference in the parity of the mode leads to a tiny overlap integral, as shown in the schematic of Fig. $5 f$.

An important consideration for the SHG from TMDC metasurfaces is the mutual arrangement of the $\mathrm{MoS}_{2}$ crystalline orientation with respect to the metasurface lattice and the incident polarisation. This arrangement is depicted in Fig. 6a, where the lab-frame is oriented along the metasurface lattice, $\hat{\mathbf{p}}$ is the pump polarisation unit vector, $\hat{\mathbf{a}}$ is the armchair direction unit vector, $\left(_{1}, \hat{\ell}_{2}\right)$ are the primitive vectors of the metasurface lattice, $(\hat{\mathbf{x}}, \hat{\mathbf{y}})$ are the lab-frame axes, $\phi_{a}$ is the angle between the armchair direction and the lab-frame, and $\phi_{p}$ is the angle between the pump polarisation and lab-frame. The SHG efficiency from the TMDC metasurface, $\eta_{\mathrm{SHG}}$, is a function of both $\phi_{p}$ and $\phi_{a}$. In the numerical simulations, we fix $\phi_{a}=0$ and evaluate the SHG conversion efficiency as a function of the pump wavelength and the angle of polarisation $\phi_{p}$ in the forward (Fig. 6) and backward (see Supplementary Fig. 8 of Supplementary Information) directions.

This polarisation/wavelength-resolved analysis confirms the conclusion that when the $\mathrm{SH}$ wavelength is either at MQ or MD QNMs (magenta and black dashed vertical lines in Fig. 6b, respectively), the SHG efficiency is maximised. In this situation, there are six peaks of equal strength when the incident polarization is varied. These peaks correspond to polarisations perpendicular to the armchair direction of the $\mathrm{MoS}_{2}$ (magenta and black plots, labelled $\lambda_{\mathrm{MQ}}$ and $\lambda_{\mathrm{MD}}$, respectively in Fig. 6c), meaning that there is only one radiating $\mathrm{SH}$ mode because of an optimal overlap of $\mathrm{SH}$ light with the eigenfields of magnetic modes. On the other hand, when the SH wavelength is near the $\mathrm{ED}$ or $\mathrm{ED}^{*}$ wavelengths (red and blue dashed vertical lines in Fig. $6 \mathrm{~b}$ ), the ED mode is weakly coupled to the SH source and therefore, the $\mathrm{SH}$ originates from the interference of the three QNMs, rather than only the ED mode. In fact, depending on the exact wavelength near the $\mathrm{ED}$, one can have stronger dominance of the ED mode (red plot, labelled $\lambda_{\mathrm{ED}}$ in Fig. 6c, with four stronger peaks and two weaker peaks) or suppression of the ED mode (blue plot, labelled $\lambda_{\mathrm{ED}}{ }^{*}$ in Fig. 6c, with two stronger peaks and four weaker peaks). It is worth mentioning that the coupling of the SH light to the electric and magnetic modes depends upon the shape, location of the nonlinear current source and symmetry of the meta-atoms, which eventually modulate the shape of the polarization-resolved SHG. As such, the SH emission dramatically depends on the tapering angle of the meta-atoms, which in the experiment is fixed by the fabrication. The simulated SHG in meta-atoms of two other tapering angles, e.g. conical meta-atoms and straight cylindrical meta-atoms are depicted for completeness in Supplementary Fig. 9 of the Supplementary Information.

To verify the polarization dependence of the SHG from TMDC metasurfaces, we have also performed experiments on polarisation resolved SHG in forward direction at the pump wavelength of $1550 \mathrm{~nm}$. The measured polarization-resolved SHG is shown in Fig. 6d. It demonstrates sixfold rotational symmetry, when $\phi_{p}$ varies from 0 to $360^{\circ}$. There is a very good agreement between our experimental (Fig. 6d) and simulated data (Fig. 6b, c). A comparison of the simulated and experimental results reveals that, in the experiment, the actual armchair direction is at $\phi_{a} \approx-30^{\circ}$. Indeed, when in simulations we set $\phi_{a}=-30^{\circ}$, we obtain excellent agreement with the experimental polarizationresolved SHG, as shown in Fig. 6d, e. For the chosen pump wavelength generating $\mathrm{SH}$ in the vicinity of the ED mode, instead of six peaks of equal amplitude, in Fig. 6d, e we can observe four stronger peaks.

Finally, the most intriguing feature of the presented $\mathrm{MoS}_{2}$ metasurface is the capability to tune the directionality of the $\mathrm{SH}$ emissions and switch the emission between forward and backward direction. While SH emission has been tested for various dielectric metasurfaces ${ }^{16,17,23,24,55,56}$, no directionality-switching capability has been reported so far. In ref. ${ }^{31}$ switching of the $\mathrm{SH}$ emission has been observed for a single (110) GaAs nanoantenna. However, no metasurfaces of such nanoantennas have yet been realised to date. Furthermore, all metasurfaces to date that are resonant at the fundamental wave have multiple diffraction orders at the SH wave, which would not allow for pure forward to backward switching of the SH emissions. Here, we demonstrate a dielectric metasurface with forward to backward continuous tunability of the $\mathrm{SH}$ emissions. This property is a direct consequence of the position of the nonlinear sources and the interference of the QNMs in the far-field. Importantly, to tune the SH directionality, no physical change in the structure or complicated geometry is required. The tuning can be achieved by varying the input wavelength or polarisation.

To quantify such tuning of the $\mathrm{SH}$ emission, we define the $\mathrm{SH}$ directionality in terms of the relative power of the SH emitted in forward (F) direction as $D_{F}=\frac{P_{F}^{\mathrm{SH}}}{P_{F}^{\mathrm{SH}}+P_{B}^{\mathrm{SH}}}$ and in backward

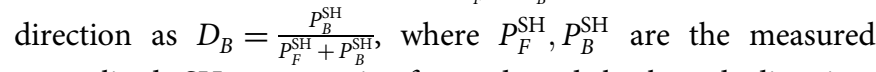
normalised $\mathrm{SH}$ powers in forward and backward direction, respectively. In Fig. 7a, we show the experimentally measured $\mathrm{SH}$ directionalities, $D_{F}$ in blue and $D_{B}$ in red, as a function of the pump wavelength, for $\phi_{p}=0$ and $\phi_{a}=-30^{\circ}$. In the bar charts, 0.5 is the threshold value, which defines the switching of $\mathrm{SH}$ emissions from forward to backward direction when $D_{F}<0.5$ and $D_{B}>0.5$. In Fig. $7 \mathrm{a}$, the SH directionality switching point can be observed around $740 \mathrm{~nm}$. In Fig. $7 \mathrm{a}$, the $x$-axis scaling (representing SH wavelengths) corresponds to the selected fundamental wavelengths, where our OPO shows stable performance in the spectral range from 1400 to $1600 \mathrm{~nm}$. Figure $7 \mathrm{~b}$ depicts the measured SH directionality, $D_{F}$ and $D_{B}$, at switching 
a

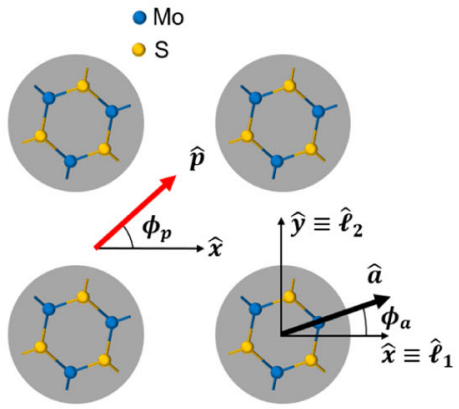

b

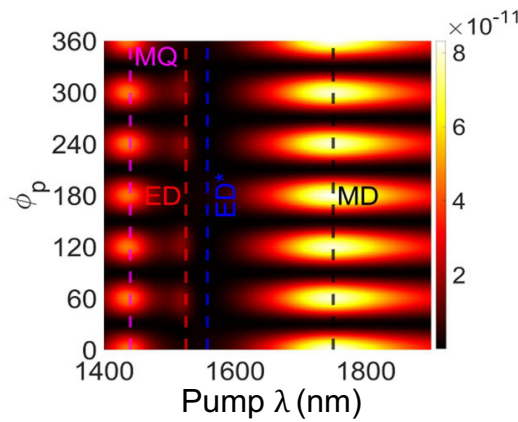

C

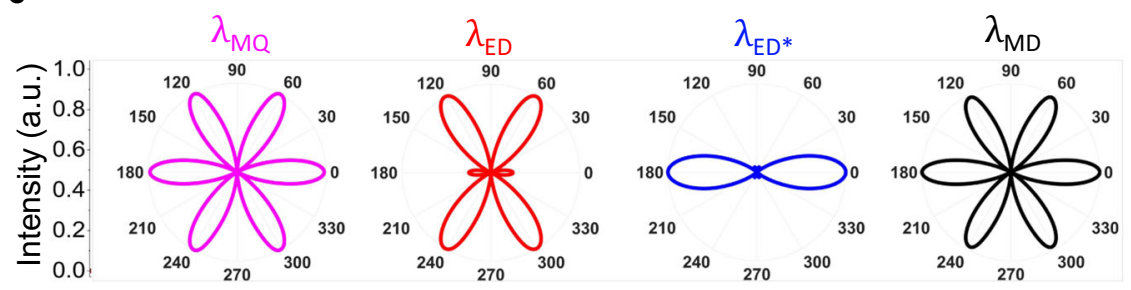

d

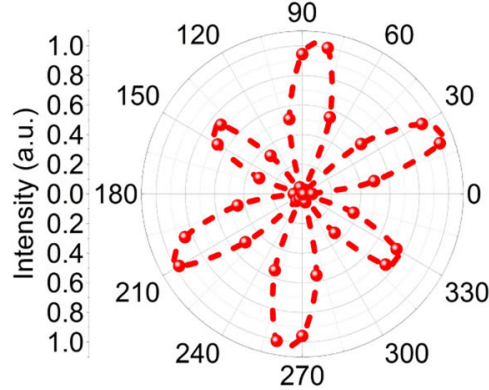

e

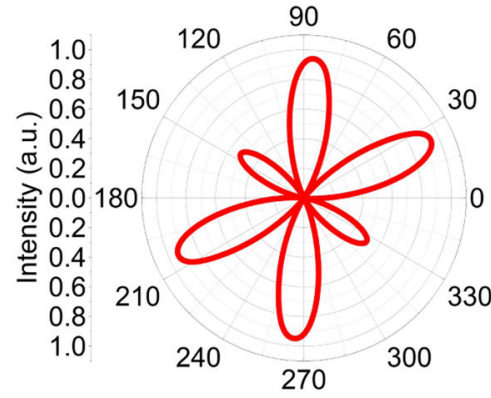

Fig. 6 Polarisation-resolved SHG in a TMDC metasurface. a Schematic of the crystal orientation of the MoS $_{2}$ with respect to the metasurface lattice (shown by primitive vectors $\hat{\ell}_{1}$ and $\hat{\ell}_{2}$ ) and incident polarisation ( $\hat{\boldsymbol{p}}$ : polarisation unit vector). The lab frame axis and armchair direction unit vectors are shown by $\hat{\boldsymbol{x}}, \hat{\boldsymbol{y}}$ and $\hat{\boldsymbol{a}}$, respectively. $\mathbf{b}$ Simulated polarisation resolved SHG, in the forward direction, as a function of angle between pump polarisation and lab frame $\phi_{p}$ and pump wavelength when angle between armchair direction and lab frame $\phi_{a}=0$. The vertical dashed lines represent the spectral positions of the magnetic quadrupole (MQ), electric dipole (ED), the point in close vicinity of electric dipole (ED*), and magnetic dipole (MD). c Polarisation resolved SHG as a function of $\phi_{p}$ (when $\phi_{a}=0$ ), at the wavelengths marked MQ, ED, ED* and MD. $\mathbf{d}$ Measured polarisation resolved SHG as a function of $\phi_{p}$ (when $\phi_{a}=30^{\circ}$ ) of metasurface $\mathrm{C}$ at ED. e Simulated polarisation resolved SHG as function of $\phi_{p}$ (when $\phi_{a}=30^{\circ}$ ) of metasurface $\mathrm{C}$ at ED.
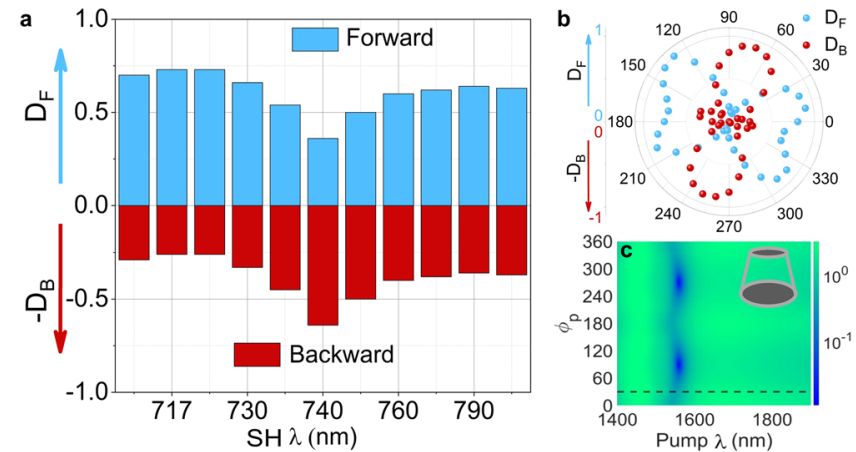

Fig. 7 Directionality switching of the second harmonic emission. a Measured $\mathrm{SH}$ directionality in terms of the relative power of the $\mathrm{SH}$ emissions in forward direction $D_{F}$ and in backward direction $D_{B}$ directions as a function of the second harmonic wavelength, when angle between pump polarisation and lab frame $\phi_{p}=0^{\circ}$. b Measured forward and backward SH directionality at switching wavelength, $1480 \mathrm{~nm}$, as a function of angle between pump polarisation and lab frame $\phi_{p}$ when angle between armchair direction and lab frame $\phi_{a}=-26^{\circ}$. c Simulated directionality mapping, as ratio of forward and backward $\mathrm{SH}$ emissions $\left(P_{F}^{\mathrm{SH}} / P_{B}^{\mathrm{SH}}\right)$ from the truncated cone (as shown in the inset) metasurface, as a function of pump wavelength and $\phi_{p}$, when $\phi_{a}=0^{\circ}$. point as a function of $\phi_{p}$. We further complement our measurements by numerical simulations and in Fig. 7c we show the simulated $\mathrm{SH}$ directionality mapping, $\left(P_{F}^{\mathrm{SH}} / P_{B}^{\mathrm{SH}}\right)$, as function of pump wavelength and $\phi_{p}$ for $\phi_{a}=0$. The measured data in Fig. 7a corresponds to the horizontal dashed line in Fig. 7c. The switching point of the $\mathrm{SH}$ directionality as a function of $\phi_{p}$ is observed for two values $\phi_{p} \cong 80^{\circ}$ and $\cong 260^{\circ}$. In Fig. $7 \mathrm{c}$, it can also be seen that the $\mathrm{SH}$ directionality switching occurs in the close vicinity of the ED mode, which can be triggered either as a function of the pump wavelength or polarisation. In contrast, the directionality of the $\mathrm{SH}$ emission is symmetric at the MQ and MD resonances. A good agreement can be observed between the experimental and simulated directionality data. The difference between the measured and simulated data might be because of the directionality being sensitive to the exact shape of the fabricated $\mathrm{MoS}_{2}$ meta-atoms, such as the tapering angle and the surface roughness.

To illustrate, the effect of the tapering on the switching of the $\mathrm{SH}$ directionality in Fig. 8 we have simulated the SH directionality (see also Supplementary Figure 10 of Supplementary Information) when the $\mathrm{MoS}_{2}$ meta-atom has the shape of a straight vertical cylinder (no sidewall tapering). The data in Fig. 8a corresponds to the horizontal dash line in Fig. 8c. It may be seen that in this cylindrical geometry, one can achieve a nearly 

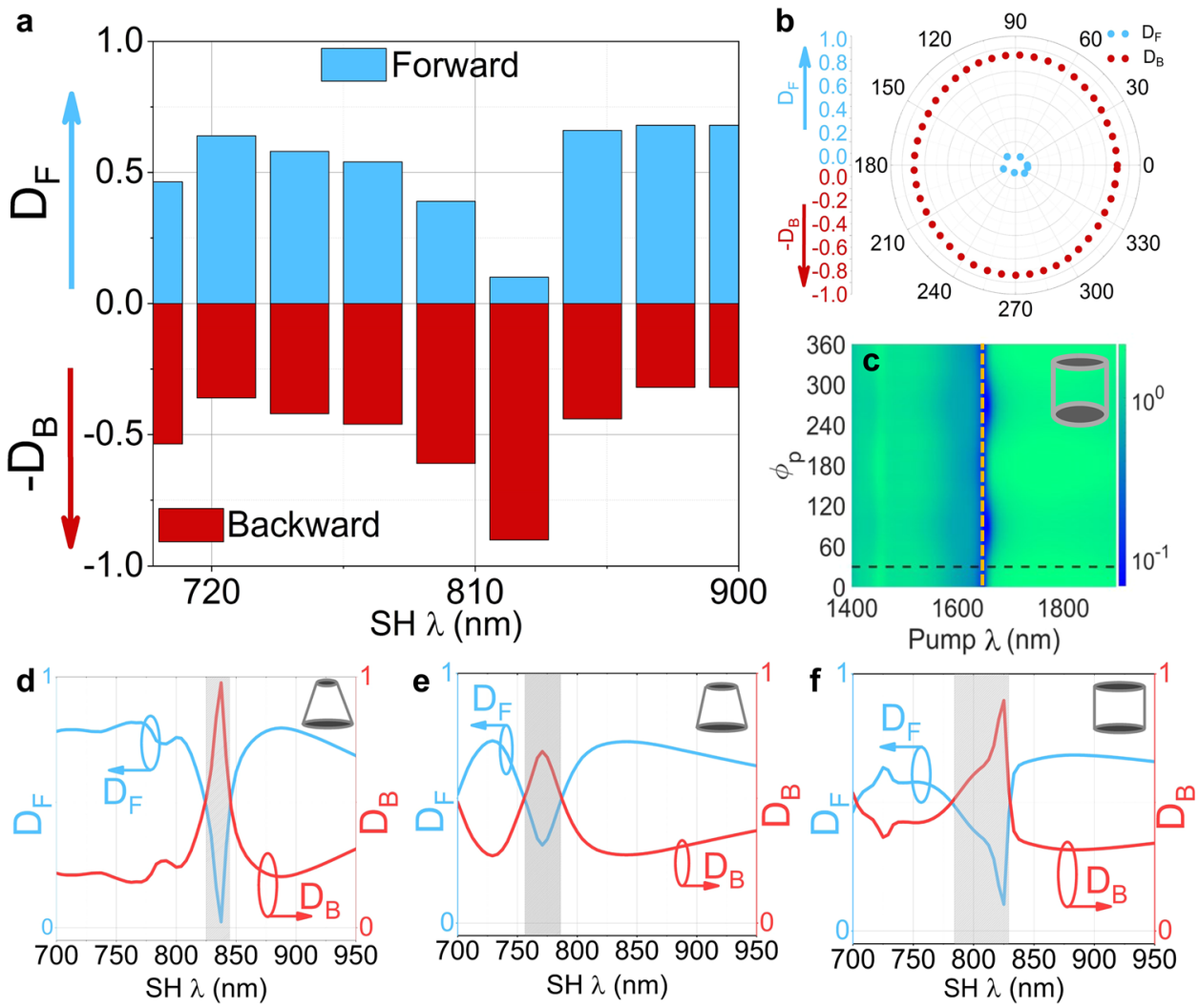

Fig. 8 Tapering angle effect on directionality switching of the second harmonic emission. a Simulated SH directionality in terms of the relative power of the $\mathrm{SH}$ emissions in forward direction $D_{F}$ and in backward direction $D_{B}$ of $\mathrm{MoS}_{2}$ metasurface with straight vertical walls of cylindrical meta-atoms, as a

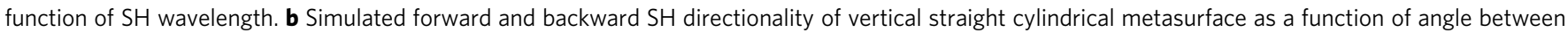
pump polarisation and lab frame $\phi_{\mathrm{p}}$ at a pump wavelength of $\cong 1650 \mathrm{~nm}$. c Simulated directionality mapping, as ratio of forward and backward SH emissions $\left(P_{F}^{S H} / P_{B}^{S H}\right)$ of a straight cylindrical (as shown in the inset) $M_{0} S_{2}$ metasurface, as function of pump wavelength and $\phi_{p}$, when angle between

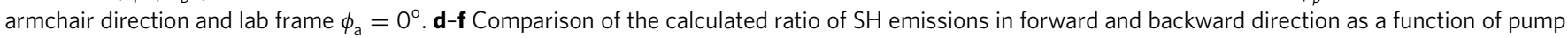
wavelength. d Cone meta-atom. e Truncated cone meta-atom. $\mathbf{f}$ Vertical cylinder. The shaded area shows the switching point of the directionality.

complete switching of the $\mathrm{SH}$ emission from forward $(\sim 725 \mathrm{~nm})$ to backward $(\sim 825 \mathrm{~nm})$ direction, as shown in Fig. 8a. Such nonlinear switching is useful for application in nonlinear mirrors and laser mode-locking ${ }^{34}$. In addition, in the case of the vertical straight cylindrical metasurface, the $\mathrm{SH}$ directionality as function of $\phi_{p}$ shows no switching at the wavelength of $825 \mathrm{~nm}$. This polarization dependence of the directionality is shown in Fig. 8b and corresponds to the vertical dash-dotted line in Fig. 8c.

Furthermore, in Fig. $8 \mathrm{~d}-\mathrm{f}$ we show the effect of the tapering angle on the second harmonic directionality. Three shapes with three different tapering angles of the meta-atoms have been calculated and compared in Fig. $8 \mathrm{~d}-\mathrm{f}$. It is seen that the contrast of the unidirectional emission can be controlled with the tapering angle of the meta-atoms. However, the tapering angle also affects the switching-point wavelength and the spectral bandwidth of the switching. These changes are likely due to the spectral shifts of the resonances. In our experimental case, the switching ratio is lower than in the other two cases, however, it is also spectrally broader and bell-shaped, which has improved the robustness of this condition to experimental imperfections.

\section{Discussions}

In summary, we have demonstrated the directional nonlinear harmonic generation in a high-refractive-index single-crystal vdW $\mathrm{MoS}_{2}$. The vdW interactions between the TMDC layers offer a unique opportunity to realise such metasurfaces on any smooth and transparent substrate, thus one can excite and detect the $\mathrm{SH}$ and $\mathrm{TH}$ signals from each side of the metasurface. We have experimentally and theoretically demonstrated that by changing the aspect ratio of the truncated-cone meta-atoms, one can tune the induced resonances of the metasurface and provide direct control over the second- and third-order nonlinear generation. As compared to other dielectric and semiconductor metasurfaces the use of the high-index TMDCs allows to design of highly subwavelength resonators and therefore to construct nonlinear metasurfaces with single-beam emission at the harmonic waves. In this sub-diffractive regime, only a zero diffraction order is emitted from the metasurface, which makes it act as a homogeneous ultra-thin nonlinear crystal but with enhanced conversion efficiency. Most importantly, the second-harmonic emission can be tuned from forward to backward direction, controlled by wavelength, incident polarisation and metasurface geometry. This tunable unidirectional nonlinear emission facilitates novel applications in nonlinear light sources.

In addition, we have employed Mie-resonances at the harmonic wavelengths as a versatile tool to control both SHG and THG at the nanoscale. The choice of resonances at the harmonic wavelengths further makes our metasurfaces deeply subwavelength for both the second and third harmonic waves. Our work aims to demonstrate the fundamental physical phenomena on the nonlinear emission in TMDC metasurfaces and as such is able to unveil the origin of the quadratic nonlinearities in TMDC metasurfaces. These stem from the broken crystalline symmetry at the top and bottom layer of the meta-atoms. This feature is 
Table 1 Performance comparison of the $\mathrm{MoS}_{2}$ metasurface with other high index dielectric metasurfaces.

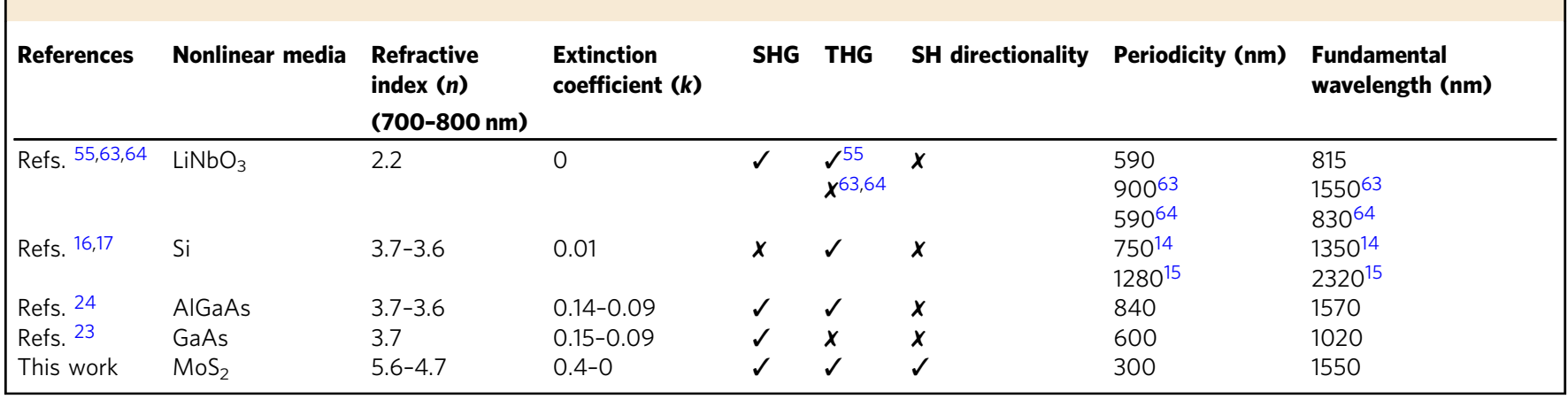

distinct to the harmonic generation by surface nonlinearities in other bulk non-centrosymmetric material, such as silicon and results in more than two orders of magnitude higher SHG efficiency with respect to silicon metasurfaces. We note that while our metasurface design does not aim at reaching record-breaking conversion efficiencies, it is possible to applied multiple strategies for increasing the efficiency of both SHG and THG. These include designs with higher quality resonances, such as in the case of bound-state in the continuum ${ }^{57}$.

The comparison of the performance of our TMDC metasurface with other existing works, in terms of its high index and $\mathrm{SH}$ directionality switching, has been presented in Table 1 . The cross symbol in Table 1 refers to the lack of SHG/THG measurement or no SHG/THG in those references. We note that while the nonlinear emission from monolayer TMDCs coupled to plasmonic ${ }^{58,59}$ or dielectric metasurfaces ${ }^{60}$ have also been recently studied, our TMDC metasurface offers several fundamental advantages. These include the ability to simultaneously enhance the SHG and THG processes, offering a significantly higher interaction volume for the THG. For the case of SHG, it further offers the ability to switch the directionality of $\mathrm{SH}$ emission between forward and backward direction, which so far has not been demonstrated in other metasurface systems. Importantly, this intriguing feature has been achieved all-optically: there is no need to apply physical changes to the metasurface and can be dynamically controlled by varying the pump wavelength or polarisation. We have experimentally proven this $\mathrm{SH}$ directionality switching and have theoretically shown that the contrast of this switching can be further enhanced for the case of vertical cylinders, thereby virtually acting as a mirror for the SH light.

\section{Methods}

Device fabrication. We fabricated multiple arrays of $\mathrm{MoS}_{2}$ nanoresonators $\left(\mathrm{MoS}_{2}\right.$ metasurfaces) on a sapphire $\left(\mathrm{Al}_{2} \mathrm{O}_{3}\right)$ substrate. To achieve the pristine quality of the sample, we mechanically exfoliated vdW material $\mathrm{MoS}_{2}$ onto the substrate. The thickness of the transferred flakes was then measured by a surface profilometer. After cleaning, we spin-coated a single layer of the ZEP positive resist followed by a thin layer of conductive polymer (to dissipate the charges during the lithography process) on the selected flake. The flake was subsequently patterned into nanoresonators using EBL. After developing the sample, we deposit an $80 \mathrm{~nm}$ thick layer of the $\mathrm{Al}$ (for dry etching mask), using an e-beam evaporator. The truncated cone nano-pillars were then dry-etched by employing inductive-coupled plasma of $\mathrm{SF}_{6}+\mathrm{CHF}_{3}+\mathrm{Ar}$. and then $\mathrm{Al}$ caps from the top of the truncated-cone pillars removed by a lift-off process. Finally, we employed SEM to examine the quality and dimensions of the $\mathrm{MoS}_{2}$ metasurfaces.

Numerical calculations. To theoretically describe our experimental results, first, we performed FDTD simulations using the Lumerical FDTD tool for the manifestation of mode properties in the $\mathrm{SH}$ spectrum $(700-900 \mathrm{~nm})$. We used periodic boundary conditions along with normal-incidence plane-wave illumination from either side (the air or substrate side) of the metasurface and calculated the linear spectra of the metasurface from both sides. The data presented in the paper corresponds to the plane-wave illumination from the airside to match our experimental arrangement. We used the refractive index and extinction coefficient of the $\mathrm{MoS}_{2}$ bulk from a work presented in ref. ${ }^{61}$ (see Supplementary Fig. 1). For the nonlinear simulations, we modelled the truncated cone meta-atoms in COMSOL multiphysics. We employed full-wave nonlinear simulations in COMSOL (finiteelement method) to extract the $\mathrm{SH}$ and $\mathrm{TH}$ efficiency in the range of pump wavelength. In simulations, the pump is incident from the airside (as in the experiment), $s$-polarised in the $x$-direction $\left(\phi_{p}=0\right)$. Moreover, we use pump intensity of $1 \mathrm{GWcm}^{-2}$ and $\chi^{2}\left(\mathrm{MoS}_{2}\right)$ tensor value of $3 \mathrm{~nm}^{2} \mathrm{~V}^{-1}$ in agreement with experimental measurements presented in ref. ${ }^{62}$. To gain a deeper insight into the physics of SH peaks, we employed QNMs theory and solved the complex eigenvalue problem with a finite-element method (COMSOL).

\section{Data availability}

The $\mathrm{MoS}_{2}$ dispersion data used in this study is provided in the Supplementary Table 2.

Received: 19 January 2021; Accepted: 23 August 2021; Published online: 22 September 2021

\section{References}

1. De Angelis, C., Leo, G. \& Neshev, D. N. Nonlinear Meta-Optics. (CRC Press, 2020).

2. Keren-Zur, S., Michaeli, L., Suchowski, H. \& Ellenbogen, T. Shaping light with nonlinear metasurfaces. Adv. Opt. Photonics 10, 309-353 (2018).

3. Kauranen, M. \& Zayats, A. V. Nonlinear plasmonics. Nat. Photonics 6, 737-748 (2012)

4. Aouani, H. et al. Multiresonant broadband optical antennas as efficient tunable nanosources of second harmonic light. Nano Lett. 12, 4997-5002 (2012).

5. Celebrano, M. et al. Mode matching in multiresonant plasmonic nanoantennas for enhanced second harmonic generation. Nat. Nanotechnol. 10, 412-417 (2015)

6. Klein, M. W., Enkrich, C., Wegener, M. \& Linden, S. Second-harmonic generation from magnetic metamaterials. Science 313, 502-504 (2006).

7. Krasnok, A., Tymchenko, M. \& Alù, A. Nonlinear metasurfaces: a paradigm shift in nonlinear optics. Mater. Today 21, 8-21 (2018).

8. Lee, J. et al. Giant nonlinear response from plasmonic metasurfaces coupled to intersubband transitions. Nature 511, 65-69 (2014).

9. Rosencher, E. et al. Quantum engineering of optical nonlinearities. Science 271, 168-173 (1996).

10. Kuznetsov, A. I., Miroshnichenko, A. E., Brongersma, M. L., Kivshar, Y. S. \& Luk'yanchuk, B. Optically resonant dielectric nanostructures. Science 354, https://doi.org/10.1126/science.aag2472 (2016).

11. Neshev, D. \& Aharonovich, I. Optical metasurfaces: new generation building blocks for multi-functional optics. Light Sci. Appl. 7, 58 (2018).

12. Smirnova, D. \& Kivshar, Y. S. Multipolar nonlinear nanophotonics. Optica 3, 1241-1255 (2016).

13. Kivshar, Y. All-dielectric meta-optics and non-linear nanophotonics. Natl Sci. Rev. 5, 144-158 (2018).

14. Sain, B., Meier, C. \& Zentgraf, T. Nonlinear optics in all-dielectric nanoantennas and metasurfaces: a review. Adv. Photonics 1, 024002 (2019).

15. Shcherbakov, M. R. et al. Enhanced third-harmonic generation in silicon nanoparticles driven by magnetic response. Nano Lett. 14, 6488-6492 (2014).

16. Yang, Y. et al. Nonlinear fano-resonant dielectric metasurfaces. Nano Lett. 15 7388-7393 (2015)

17. Liu, H. et al. Enhanced high-harmonic generation from an all-dielectric metasurface. Nat. Phys. 14, 1006-1010 (2018)

18. Grinblat, G., Li, Y., Nielsen, M. P., Oulton, R. F. \& Maier, S. A. Efficient third harmonic generation and nonlinear subwavelength imaging at a higher-order anapole mode in a single germanium nanodisk. ACS Nano 11, 953-960 (2017) 
19. Ghirardini, L. et al. Polarization properties of second-harmonic generation in AlGaAs optical nanoantennas. Opt. Lett. 42, 559-562 (2017).

20. Carletti, L., Locatelli, A., Stepanenko, O., Leo, G. \& De Angelis, C. Enhanced second-harmonic generation from magnetic resonance in AlGaAs nanoantennas. Opt. Express 2, 1007-1012 (2015).

21. Gili, V. F. et al. Monolithic AlGaAs second-harmonic nanoantennas. Opt. Express 24, 15965-15971 (2016).

22. Carletti, L. et al. Controlling second-harmonic generation at the nanoscale with monolithic AlGaAs-on-AlOx antennas. Nanotechnology 28, https:// doi.org/10.1088/1361-6528/aa5645 (2017).

23. Liu, S. et al. Resonantly enhanced second-harmonic generation using III-V semiconductor all-dielectric metasurfaces. Nano Lett. 16, 5426-5432 (2016)

24. Liu, S. et al. An all-dielectric metasurface as a broadband optical frequency mixer. Nat. Commun. 9, 1-6 (2018).

25. Koshelev, K. et al. Subwavelength dielectric resonators for nonlinear nanophotonics. Science 367, 288-292 (2020).

26. Camacho-Morales, R. et al. Nonlinear generation of vector beams from AlGaAs nanoantennas. Nano Lett. 16, 7191-7197 (2016).

27. Löchner, F. J. F. et al. Polarization-dependent second harmonic diffraction from resonant GaAs metasurfaces. ACS Photonics 5, 1786-1793 (2018).

28. Vabishchevich, P. P. et al. Enhanced second-harmonic generation using broken symmetry III-V semiconductor fano metasurfaces. ACS Photonics 5, 1685-1690 (2018).

29. Rocco, D. et al. Vertical second harmonic generation in asymmetric dielectric nanoantennas. IEEE Photonics J. 12, 1-7 (2020).

30. Sautter, J. D. et al. Tailoring second-harmonic emission from (111)-GaAs nanoantennas. Nano Lett. 27, 30909-30918 (2019).

31. $\mathrm{Xu}, \mathrm{L}$. et al. Forward and backward switching of nonlinear unidirectional emission from GaAs nanoantennas. ACS Nano 14, 1379-1389 (2020).

32. Marino, G. et al. Zero-order second harmonic generation from AlGaAs-oninsulator metasurfaces. ACS Photonics 6, 1226-1231 (2019).

33. Garmire, E. Nonlinear optics in daily life. Opt. Express 21, 30532-30544 (2013).

34. Stankov, K. A. \& Jethwa, J. A new mode-locking technique using a nonlinear mirror. Opt. Commun. 18, 12663-12668 (1988).

35. Yang, J. et al. Atomically thin optical lenses and gratings. Light Sci. Appl. 5, e16046 (2016).

36. van de Groep, J. et al. Exciton resonance tuning of an atomically thin lens. Nat. Photonics 14, 426-430 (2020).

37. Mak, K. F. \& Shan, J. Photonics and optoelectronics of $2 \mathrm{D}$ semiconductor transition metal dichalcogenides. Nat. Photonics 10, 216-226 (2016).

38. Verre, R. et al. Transition metal dichalcogenide nanodisks as high-index dielectric Mie nanoresonators. Nat. Nanotechnol. 14, 679-683 (2019).

39. Liu, C. H. et al. Ultrathin van der Waals Metalenses. Nano Lett. 18, 6961-6966 (2018).

40. Green, T. et al. Optical material anisotropy in high-index transition metal dichalcogenide Mie nanoresonators. Optica 7, 680-686 (2020).

41. Busschaert, S. et al. TMDC resonators for second harmonic signal enhancement. ACS Photonics 7, 2482-2488 (2020).

42. Dasgupta, A., Yang, X. \& Gao, J. Nonlinear beam shaping with binary phase modulation on patterned WS2 monolayer. ACS Photonics 7, 2506-2514 (2020).

43. Löchner, F. J. F. et al. Controlling second-harmonic diffraction by nanopatterning $\mathrm{MoS}_{2}$ monolayers. Opt. Express 27, 35475-35484 (2019).

44. Dasgupta, A., Gao, J. \& Yang, X. Atomically thin nonlinear transition metal dichalcogenide holograms. Nano Lett. 19, 6511-6516 (2019).

45. Tribelsky, M. I. \& Miroshnichenko, A. E. Giant in-particle field concentration and Fano resonances at light scattering by high-refractive-index particles. Phys. Rev. A 93, 053837 (2016)

46. Staude, I. et al. Tailoring directional scattering through magnetic and electric resonances in subwavelength silicon nanodisks. ACS Nano 7, 7824-7832 (2013).

47. Gigli, C. et al. Quasinormal-mode non-hermitian modeling and design in nonlinear nano-optics. ACS Photonics 7, 1197-1205 (2020).

48. Lalanne, P., Yan, W., Vynck, K., Sauvan, C. \& Hugonin, J. P. Light interaction with photonic and plasmonic resonances. Laser Photonics Rev. 12, 1700113 (2018).

49. Li, Y. et al. Probing symmetry properties of few-layer MoS2 and h-BN by optical second-harmonic generation. Nano Lett. 13, 3329-3333 (2013).

50. Säynätjoki, A. et al. Ultra-strong nonlinear optical processes and trigonal warping in MoS2 layers. Nat. Commun. 8, 893 (2017).

51. Rosa, H. G. et al. Characterisation of the second- and third-harmonic optical susceptibilities of atomically thin tungsten diselenide. Sci. Rep. 8, 10035 (2018).

52. Hessel, A. \& Oliner, A. A. A new theory of wood's anomalies on optical gratings. Appl. Opt. 4, 1275-1297 (1965).

53. Metzger, B. et al. Strong enhancement of second harmonic emission by plasmonic resonances at the second harmonic wavelength. Nano Lett. 15 3917-3922 (2015).

54. Bar-David, J. \& Levy, U. Nonlinear diffraction in asymmetric dielectric metasurfaces. Nano Lett. 19, 1044-1051 (2019).
55. Carletti, L. et al. Second harmonic generation in monolithic lithium niobate metasurfaces. Opt. Express 27, 33391-33398 (2019).

56. Sung, J., Lee, G. Y., Choi, C., Hong, J. \& Lee, B. Single-layer bifacial metasurface: full-space visible light control. Adv. Opt. Mater. 7, 1801748 (2019).

57. $\mathrm{Xu}, \mathrm{L}$. et al. Dynamic nonlinear image tuning through magnetic dipole quasiBIC ultrathin resonators. Adv. Sci. 6, 1802119 (2019).

58. $\mathrm{Hu}, \mathrm{G}$. et al. Coherent steering of nonlinear chiral valley photons with a synthetic Au-WS2 metasurface. Nat. Photonics 13, 467-472 (2019).

59. Hong, X. et al. Structuring nonlinear wavefront emitted from monolayer transition-metal dichalcogenides. Research 2020, 9085782 (2020).

60. Yuan, Q. et al. Second harmonic and sum-frequency generations from a silicon metasurface integrated with a two-dimensional material. ACS Photonics 6, 2252-2259 (2019).

61. Beal, A. R. \& Hughes, H. P. Kramers-Kronig analysis of the reflectivity spectra of 2H-MoS2, 2H-MoSe2 and 2H-MoTe2. J. Phys. C Solid State Phys. 12, 5 (1979).

62. Malard, L. M., Alencar, T. V., Barboza, A. P. M., Mak, K. F. \& de Paula, A. M. Observation of intense second harmonic generation from $\mathrm{MoS}_{2}$ atomic crystals. Phys. Rev. B 87, 20 (2013).

63. Fedotova, A. et al. Second-harmonic generation in resonant nonlinear metasurfaces based on lithium niobate. Nano Lett. 20, 8608-8614 (2020).

64. Carletti, L. et al. Steering and encoding the polarization of the second harmonic in the visible with a monolithic LiNbO3 metasurface. ACS Photonics 8, 731-737 (2021)

\section{Acknowledgements}

This work was supported by The Punjab Educational Endowment Fund (PEEF) Pakistan, by the Australian Research Council through Centres of Excellence (CE20010001, CE170100012), Discovery Projects (DP190101559, DP180103238 and DP200101353) and ARC Early Career Researcher Award (DE190100430) programs, as well as the UK Research and Innovation Future Leaders Fellowship (MR/T040513/1). We acknowledge the use of the ACT node of the Australian National Fabrication Facility (ANFF).

\section{Author contributions}

M.N., M.R., Y.L., and D.N.N. conceived the idea. M.N., D.d.C., M.R. and D.N.N. designed the samples. M.N., K.Z.K, Y.L., and M.R. fabricated the samples. J.Y. and M.N cunducted the experiments. D.d.C., C.d.A. and A.E.M performed the analytical modelling and numerical simulations. M.N., J.Y., D.d.C, M.R., C.d.A., A.E.M., Y.L. and D.N.N analysed the experimental and theoretical results. All authors contributed to discussions and writing the paper.

\section{Competing interests}

The authors declare no competing interests.

\section{Additional information}

Supplementary information The online version contains supplementary material available at https://doi.org/10.1038/s41467-021-25717-x.

Correspondence and requests for materials should be addressed to Domenico de Ceglia, Andrey E. Miroshnichenko, Yuerui Lu or Dragomir N. Neshev.

Peer review information Nature Communications thanks Byoungho Lee and the other, anonymous, reviewer(s) for their contribution to the peer review of this work.

Reprints and permission information is available at http://www.nature.com/reprints

Publisher's note Springer Nature remains neutral with regard to jurisdictional claims in published maps and institutional affiliations.

Open Access This article is licensed under a Creative Commons Attribution 4.0 International License, which permits use, sharing, adaptation, distribution and reproduction in any medium or format, as long as you give appropriate credit to the original author(s) and the source, provide a link to the Creative Commons license, and indicate if changes were made. The images or other third party material in this article are included in the article's Creative Commons license, unless indicated otherwise in a credit line to the material. If material is not included in the article's Creative Commons license and your intended use is not permitted by statutory regulation or exceeds the permitted use, you will need to obtain permission directly from the copyright holder. To view a copy of this license, visit http://creativecommons.org/ licenses/by/4.0/.

(c) The Author(s) 2021 